Published as: Ooms, K., De Maeyer, P., Fack, V., Van Assche, E. \& Witlox, F. (2012). Investigating the effectiveness of an Efficient Label Placement Method Using Eye Movement Data. Cartographic Journal, vol. 49 (3), 234-246.

\title{
Investigating the Effectiveness of an Efficient Label Placement Method Using Eye Movement Data
}

\author{
Ooms, K., De Maeyer, P., Fack, V., Van Assche, E., \& Witlox, F.
}

This paper focuses on improving the efficiency and effectiveness of dynamic and interactive maps in relation to the user. A label placement method with an improved algorithmic efficiency is presented. Since this algorithm has an influence on the actual placement of the name labels on the map, it is tested if this efficient algorithms also creates more effective maps: how well is the information processed by the user. We tested 30 participants while they were working on a dynamic and interactive map display. Their task was to locate geographical names on each of the presented maps. Their eye movements were registered together with the time at which a given label was found. The gathered data reveals no difference in the user's response times, neither in the number and the duration of the fixations between both map designs. The results of this study show that the efficiency of label placement algorithms can be improved without disturbing the user's cognitive map. Consequently, we created a more efficient map without affecting it's effectiveness towards the user.

\section{INTRODUCTION}

The last two decades have seen a significant growth in the number of cartographic products available on the Internet. The terms neo-geography and neo-cartography have become common in the literature (Haklay, et al., 2008; Jobst \& Döllner, 2008; Kraak \& He, 2009; Turner, 2006). These terms are closely linked with the latest technical, multimedia, and Web developments, such as Web 2.0, which combine these developments with traditional GIS, cartography, and imagery research. This evolution has greatly facilitated the accessibility and availability of geographic data, resulting in an enormous increase in web map use.

However, these new possibilities and techniques also bring new challenges. For one there is the key issue of efficiency and effectiveness of web maps. Since these terms are used in different research and application fields - each using their own definitions - there might be some confusion regarding their exact meaning. In this paper, the term efficiency, is used from an algorithmic point of view: how efficient is a certain algorithm or heuristic? One of the key factors used to measure this efficiency is the speed with which a certain algorithm can accomplish its goal (with a certain level of quality). In the case digital (Internet) maps, the 
construction of optimal algorithms and data structures to support or 'calculate' the visualization is essential. The main challenge is thus related to the interaction possibilities, because a new visualization must be calculated on the fly each time the user alters an element of the display (e.g., zoom factor, extent, colours, and layer visualization). To illustrate, if a user zooms in on a map, the extent of the map that will be displayed after the interaction changes: a smaller region is displayed but with greater detail. The algorithm must retrieve the objects within the limits of this new extent; only those objects that are linked with the correct level of detail or zoom factor are considered. The name of a country, for example, may not be displayed on a map if the user is zooms in on a little village within that country. Furthermore, users do not want to wait several seconds before a new visualization or display is generated each time they interact with it. Calculation times lasting more than one second are deemed unacceptable (Freeman, 2005). The efficiency of a web map is thus related to the fast calculation of a new display after an interaction.

The term effectiveness is in this paper used a human information processing point of view: how well can a certain person process the information presented to him? The effectiveness of a map is thus related to how well the user can process the visual information on it. Therefore, an effective map is one which can easily be processed by the user, although the information on it might be rather complex. Consequently, the effectiveness of a map is, on the one hand, inherently related to how the information is presented: the map design. On the one hand, it is also related to how users process this information. To be able to create these effective maps or map designs, it is crucial to understand how users store and process the visual information presented to them. This is in turn related to the users' cognitive processes or cognition (Harrower, 2007, Slocum, et al., 2001). Research on cognition considers different aspects of users their internal mental structures and processes related to, among others, perception, learning, memory, thinking, reasoning, and communication (Montello, 2002, 2009). As mentioned by Harrower (2007) and Slocum et al. (2001), the challenge of creating effective maps is even bigger in the case of the highly dynamic and interactive maps: the limits of the map readers' cognitive processing capabilities need to be considered.

When combining both aspects, it can be concluded that an exceptionally efficient algorithm is useless if the result is chaotic and thus difficult to process by the user. In order to create effective maps, the algorithms need to consider a number of criteria related to how the information needs to be visualised correctly. These criteria are derived from the knowledge of the users' cognitive processes and might have a negative effect on the algorithms efficiency. As a consequence, a balance has to be found between the efficiency of the algorithm on the one hand and the effectiveness of the map (or the quality of the design) on the other hand, especially when dealing with highly interactive maps.

\section{Towards Efficient Cartographic Displays}

A cartographic display consists of a number of different symbol types, each contributing to the presentation of information. Name labels are one of the most important symbol types because they contribute significantly to orienting the user within the map. Map users prefer the name 
labels on a map because less cartographic knowledge is necessary to understand the map content. These labels communicate information which cannot be presented with other types of symbols. Furthermore, labels (and their placement) can have a tremendous impact on the quality of a map because their presence informs the map user about much more than only the name of the geographic object associated to it (Barrault, 1998). They fulfil a number of essential functions related to the identification, location, orientation, and hierarchical structure of the features. They are thus essential to assisting the user in interpreting the content of the map correctly. Imhof (1975, p.128) also stated that "[...] good form and placing of type makes a good map" (Ahn \& Freeman, 1984; Wood, 2000).

Both Cuenin (1972) and Imhof (1975) proposed a limited number of basic rules to place labels on maps to create effective results. Imhof (1975, p.129) summarizes these rules as follows: "1. The names should be easily read, easily discriminated and easily and quickly located. [...]

2. The name and the object to which it belongs should be easily recognized. [...]

3. Names should disturb other map contents as little as possible. [...]

4. Names should assist directly in revealing spatial situation, territorial extent, connections, importance, and differentiation of objects.

5. Type arrangement should reflect the classification and hierarchy of objects on the map. [...]

6. Names should not be evenly dispersed over the map, nor should names be densely clustered. [...]"

Although these basic principles were defined before the popularity of digital maps, they still hold true today for the dynamic and interactive maps on the Internet. Until recently, label placement on maps was done completely manually. This was a time consuming task, which could take up to $50 \%$ or more of the whole production time of a map (Yoeli, 1972). With the evolution of technological possibilities, many studies have been devoted to automating this process. Automatic label placement only takes the first three, apparently simple, rules of Imhof (1975) into account.

However, the label placement problem - even when only considering point objects - is an NP-hard problem, which means no optimal solution for the problem can be found in an acceptable time span. Testing all possible placement options results in an exponential time complexity (Marks \& Shieber, 1991). This time complexity can be on the order of O $(4 n)$ if each point object has four candidate positions where a label can be placed, with $n$ the number of points in the dataset. In 1996, the ACM Computational Geometry Task Force identified the label placement problem as an important research field in Discrete Computational Geometry (Chazelle, et al., 1996).

Consequently, suitable heuristics and meta-heuristics are needed to find a near-optimal solution for a certain situation, such as a specific object type. Over the years, different types of heuristic approaches have been developed, among others in the field of computational geometry. A number of these optimization algorithms have been studied in the light of the label placement problem and have subsequently been adapted to improve their efficiency for this specific problem. Among the most interesting heuristics that deal with the calculation of label placement 
are: the Tabu Search Heuristic (Yamamoto, et al., 2002); Simulated Annealing (Christensen, et al., 1995; Zoraster, 1997); the Greedy Randomized Adaptive Search Procedure (Cravo, et al., 2008); Multiple Choice Integer Programming (Zoraster, 1990); and Genetic Algorithms (van Dijk, 2001). A number of solutions have also been proposed to specifically label linear objects (Edmondson, et al., 1996; Wolff, et al., 2000), and polygonal objects (Barrault, 2001). Furthermore, different applications for map displays have been taken into account in the construction of the heuristics. Automatic label placement for real-time displays has recently been given much attention (Been, et al., 2006; Mote, 2007; Yamamoto, et al., 2005; Zhang \& Harrie, 2006).

Data structures are also indispensible elements, as it is important to store and process data efficiently. This especially holds true when large amounts of labels need to be considered. The use of conflict graphs (Kakoulis \& Tollis, 2001; Wagner, et al., 2001) in a pre-processing phase allows structuring the data in a way which is surveyable, speeding up the actual calculations considerably. In this pre-processing phase, potential conflicts between the labels' candidate positions are identified and stored, using a graph. The proposal of 'sliding labels' (Strijk \& van Kreveld, 2002; van Kreveld, et al., 1999) is another example of a (data) structure that results in a more flexible selection of candidate positions in such a way that more labels can be placed. However, despite the many extensive studies related to the label placement problem, no optimal solution has yet been found. This problem is also effectively summarized by Wood (2000, p.5): "[...] no one algorithm seems to be capable of recognizing the many considerations that a skilled human cartographer is capable of making in lettering a map."

The studies discussed above mainly focus on finding efficient label placement algorithms (through the use of different heuristics and data structures), but the user side of the problem has been neglected. These heuristics are based on a number of rules concerning a good placement of the labels in relation to the user, but their final results have not been tested in an actual user study. This user side of the label placement problem is thus related to how users perceive the results of the (efficient) algorithms. A highly efficient algorithm is useless if the results it generates appear chaotic and difficult to interpret. Therefore, user studies are necessary to discover how users interpret the results from the heuristics.

van Dijk et al. (2002) have developed a method of assessing the quality of the output of the heuristics. This method employs a quality function that measures how well a certain heuristic places labels on a map. The criteria used in this function are based on the six principles proposed by Imhof (1975) and therefore have a more theoretical background. Bradstreet et al. (2005, p.1937) also described map labeling as a process in which labels should be arranged on the map "such that the result is clear enough for the map to be useful for a given user and task." They also note that 'clear enough' means the labels are readable to the user and that it is easy to identify the label with its associated site and vice versa.

In both cases, no actual users were involved in these quality assessments, making them less suitable for investigating the effectiveness of label placement in respect to the user. To truly investigate the effectiveness of the map display, a user study has to be designed. A wide range of 
techniques are available for conducting user studies, but not all techniques are equally suitable for a given problem (Downs \& Stea, 1977; Rubin \& Chisnell, 2008; van Elzakker, 2004). This paper focuses on improving the effectiveness of dynamic and interactive map displays towards the user. More specifically, the influence of different label placement options on the effectiveness of a dynamic map display is considered. In the next section, a label placement algorithm with an improved efficiency is described. A user study is constructed to test whether the user experiences any influence due to the different label layouts created by the proposed algorithm.

\section{Improved efficiency}

As mentioned in the previous section, a wide range of label placement algorithms already exists, all using a slightly different method to improve their (algorithmic) efficiency. However, they all start with the same operation: determine the objects within the current extent of the map. The most optimal location of the labels associated with these objects has to be calculated by the algorithm. How this is done exactly depends on the specific algorithm used. The 'current extent' of a digital map corresponds to the part of the map that is visible on the screen. What is more, the current extent (or view) of highly interactive maps can change very often because users can zoom, pan, etc.

When considering panning, a lot of unnecessary calculations are done (by the algorithm) after each interaction. Panning is basically a translation of the map's extent in a certain direction over a certain distance. Users often pan on a map to explore the surroundings of the current map. As a consequence, they might want to keep a rather large part of the map visible in order to orientate the newly displayed view in relation to the original one (from before the pan operation). We will call this part of the map that remains visible after the pan operation 'the overlap zone'.

This type of interaction is illustrated in Figure 1a and Figure 1b. The large blue rectangles correspond to the current view (or extent) of the map; the small rectangles represent the labels on the map. Figure 1a depicts the initial view and Figure $1 \mathrm{~b}$ the view after the interaction: a horizontal pan operation to the right. Current label placement algorithms (re)calculate an 'optimal' position for all labels within the new extent. These are the red labels in Figure 1b. Like most algorithms do, the level of preference related to a certain label position (relative to the associated point) is taken into account; the upper right position is the most preferred candidate position to place a label (Imhof, 1975). Compared to the initial view (see Figure 1a), only the dark red labels' relative positions have changed. Although all labels that are situated within the extent of the overlap zone are included in the calculations of the label placement algorithm, a more optimal position could be found for only a small number of them. A as consequence, a large number of unnecessary calculations have been done or a large number of labels have unnecessarily been included in the calculations.

Decreasing the number of labels considered by the label placement algorithm would significantly improve its efficiency. A closer look at the maps shows that most labels can remain in the same relative position, because they were already unambiguously positioned in the former view (before the interaction). Only the labels that were not visible in the former view and labels 
that could become unreadable after the interaction need to be included in the calculations. The latter group constitutes the labels located at the border of the current view, which could be clipped at the edges. Consequently, all labels that were visible in the former view and were not located near the border (and thus not in danger of being cut in half) can remain in the same relative position and should not be reconsidered by the label placement algorithm. A rather large portion of the labels will fit this category, because the panning operation is often used to explore the surroundings of a certain location. As mentioned before, the user wants to keep a part of the former view visible after the interaction to facilitate the orientation of the newly displayed area.

The outcome when using the algorithm with the improved efficiency is depicted in Figure 1c. The initial view (before the panning) still corresponds to Figure 1a. The green labels in this third picture are the ones that could safely remain in the same relative position without the danger of rendering them unreadable. Only the labels in red should be (re)considered by the label placement algorithm. The red labels on the right side of the view are those which were not yet displayed in the initial view. Since they were not yet displayed in the initial view, the algorithm has to calculate a position for them, regardless which algorithm is used. The dark red labels, were already present in the former view, but are located near the border of the current view and are thus in danger of being clipped at the edge. In this case, this is only one label at the left side of the view.

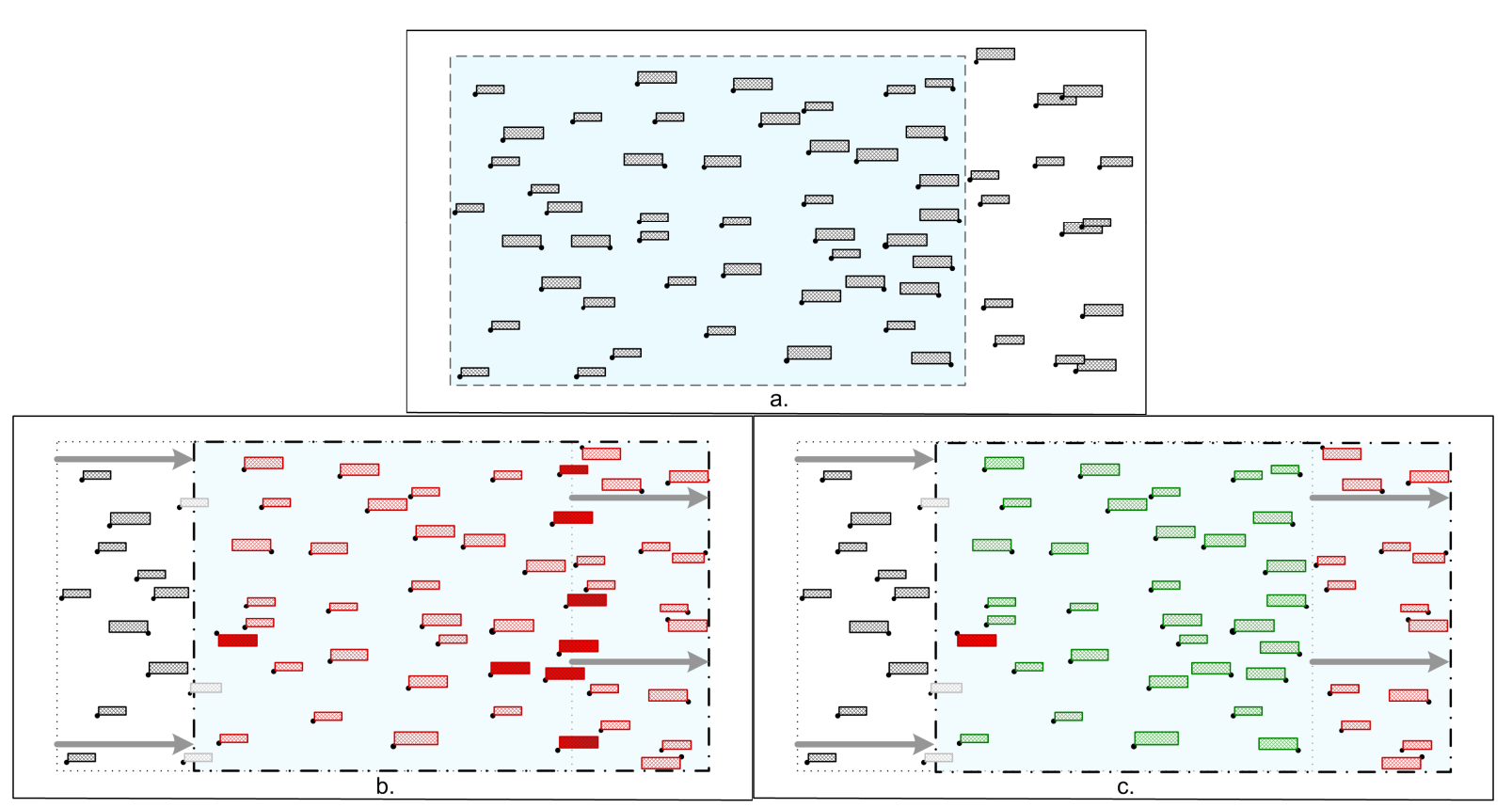

Figure 1. Illustration of the number of labels considered by the algorithms during panning

The advantage of the algorithms used today is higher level of quality of the final map. Every new view is recalculated, independently of the results of previous calculations. This means that for every view, the most optimal position for every label can be considered. However, this higher level of quality pays a price in terms of (algorithmic) efficiency because a lot of unnecessary calculations are done. The algorithm proposed in this paper has a higher level of 
efficiency since fewer calculations have to be done. Labels which were already placed in the former view are not recalculated; except for a few that otherwise would be cut in half by the border of the map.

The disadvantage of this improved algorithm (in terms of efficiency) concerns the quality of the outcome. Fewer labels are placed in their optimal position, although it would be possible to place them there. Furthermore, subsequent panning operations over a small distance decrease the quality even more: with every interaction, fewer labels will be placed in the optimal position. This lower level of quality might affect the effectiveness of the map in a negative way. In the example of Figure 1a, a number of labels are placed in the upper left corner (relative to their point object) in the initial view because otherwise they would be cut in half by the right border of the map. After the pan operation, they are still located in the upper left corner (see Figure 1c, which depicts the outcome of the proposed algorithm), although now there is space to place them in the most preferred position, which is the upper right corner (see Figure 1b, which depicts the outcome of existing algorithms). Other research concerning automatic label placement on interactive maps suggests that popping or moving of labels is also not desirable because it is distracting (Been et al., 2006). It might be more difficult for a user to orientate the map if a number of labels are in a different relative position after the interaction, because this increases complexity of the map (Harrower, 2007).

The algorithm described above improves the efficiency of the label placement algorithm, but is the outcome also beneficial towards the users? The result of the algorithm also has consequences on how the labels are displayed on the map, especially after an interaction. From the elements described above, it can be derived that the outcome of the proposed algorithm has both negative and positive elements regarding the quality of the map and thus regarding its effectiveness: fewer labels are placed in a position with a higher level of preference, but the users are less distracted after the interaction because the relative position of more labels remains the same. The goal of the current study is to investigate if the application of this algorithm might have a negative influence on the user's cognitive processes. Therefore, a suitable method, able to register changes in the user's attentive behaviour and cognitive processes while working with a dynamic medium, needs to be selected.

In this paper, we focus on the panning operations. As mentioned before, panning is an operation which is rather often executed independently from other operations - such as zooming, turning on layers and changing font sizes - to explore the surroundings of the current map view. Improving the efficiency of the label placement algorithms related to this type of interaction would therefore improve the overall processing times when (re)calculating a map's visualisation. Furthermore, turning on layers, changing font sizes, and other similar interaction possibilities are not as widely integrated in digital maps as zooming and panning. The proposed algorithm might also be beneficial when considering zooming, since some of the labels can remain in the same relative position. However, zooming on a map also influences the priority levels of the labels on the map: some labels should not be placed at a certain zoom level, whereas others might appear on that level. 


\section{Tracking effectiveness}

Nivala (2007) studied the familiarity of cartographers with usability engineering and its methods. Their results support the suitability of usability engineering for map application design, and they showed that the methods are slowly being incorporated in the design process. Consequently, research on the improvement of the effectiveness of map displays towards the user is almost nonexistent, certainly when considering the influence of label placement options on a user's cognitive processes. This lack of research is in sharp contrast with the amount of research devoted to enhancing the efficiency of label placement methods.

Duchowski (2007) and Rayner (1998) described the close link between a person's eye movements and their cognitive processes. In the past, concerns about the eye tracking method have been expressed because there is no one-to-one relation between eye movements and attentive behaviour: a person can shift his attention without moving his eyes. However, with complex stimuli (or when complex cognitive processes are triggered, causing a high cognitive load), it is more efficient to move the eyes than to move attention. As a consequence, it can safely be assumed that there is a tight link between eye movements and attentive behaviour when a person has to solve a complex task (Duchowski, 2007; Jacob \& Karn, 2003; Rayner, 1998).

During the last decade, the eye tracking method has been successfully applied to a wide range of studies concerning Human Computer Interaction (HCI) and usability research (Djamasbi, et al., 2010; Jacob \& Karn, 2003; Joachims, et al., 2005; Salvucci \& Anderson, 2001; Schiessl, et al., 2003; Zambarbieri, et al., 2008). Poole and Ball (2006, p.211) stated that this method "can help HCI researchers understand visual and display-based information processing and the factors that may impact upon the usability of system interfaces," which was also previously described in the work of Henderson and Hollingworth (1998). Moreover, Fleetwood and Byrne (2006) studied and modelled the visual search of computer users on a graphical user interface based on eye tracking data. This study has some ground in common with the experiments described in this paper. However, the study by Fleetwood and Byrne (2006) focused on the design of icons in a graphical user interface whereas the experiments described in this article are directed to the improvement of the quality of the map design for the user.

Furthermore, the eye tracking method has been introduced in the field of cartography to study, for example, the effectiveness of a new symbol design for a topographic map of Denmark (Brodersen, et al., 2001). These authors concluded that "the method as a whole is promising for future studies of map reading and map design as it reveals interesting information about a map's design, the user's behaviour, and the correlation between these" (Brodersen, et al., 2001, p.57). More recently, the eye tracking method has been integrated into studies that evaluated the effectiveness of interactive maps and small-multiple map displays (Coltekin, et al., 2009; Fabrikant, et al., 2008).

\section{STUDY DESIGN}

In the present experiment, the effectiveness of different map designs - which are a result of the application of different label placement algorithms - towards the user was tested. It was chosen to use a controlled study design in order to avoid as many (unforeseen) influencing factors as 
possible and to be able to study one element in more detail. In this experiment only one type of interaction was thus considered: panning. This choice was based on the fact that panning is possible on almost every digital map and it is an operation which is commonly executed on a map.

Each map design included in study was linked to one of two specific label placement algorithms, which are in turn linked to the efficiency of the associated algorithms. This process allowed testing whether improvements in the efficiency of the label placement algorithms - with different visual outputs - had any (negative) influence on the user's cognitive processes and thus on the effectiveness of the map display. The design of the study is described in more detail in the next sections.

\section{Participants}

Since the study has a within-user design, a group of 30 students was selected. All participants speak Dutch, have a similar educational background, and belong to the same age group (between 18 and 20 year). The participants registered to the experiment on a voluntary basis. None of them received any previous cartographic training.

\section{Task and stimuli}

To test the different map designs, a number of map stimuli were constructed. An example of such a map stimulus is presented in Figure 2. Each of these map stimuli had the same simple back ground: three areas filled with a pastel colour. On this background, point objects and their associated labels are presented. These points and the names linked to them originate from realworld locations to obtain a realistic distribution. The 20 areas selected to construct the map stimuli had to meet a number of criteria. First, the participants had to be unfamiliar with the region. Second, the region could contain only names that are rather short. Third, it had to contain a constant number of names. Fourth, the region cannot contain any well known names.

Combining these 20 areas with the two label placement options (with and without the application of the proposed algorithm) resulted in a total of 40 map stimuli. Because the duration of a user study is preferably limited, these 40 maps were divided into two sets of 20 . On ten maps of each set, the improved algorithm was applied: only labels near the border could be relocated after an interaction. On the remaining 10 maps, all labels in the map image could be relocated after the interaction. This subdivision prevented a learning effect, as the same region will not be displayed twice to a single user. All of the maps were displayed in a random order during the study.

During the display of such a map stimulus, three different time intervals could be identified. The first interval was the initial view of the map, displayed to the user for 50 seconds. Next, a horizontal pan operation was simulated. This pan operation corresponded to a translation of the map image to the right. Finally, the view after the interaction was presented until the user located all names and ended the trial. These three stages are depicted in Figure 2. The definition of the length of the initial interval was based on the results of an (unpublished) experiment, which we conducted prior to the actual experiment. The study design of this pilot study was the 
same as the one described in this paper, except that only the reaction times were measured and the time of the first interval was fixed at 90 seconds. The results of this pilot study revealed that almost all participants could find the 5 labels within 50 seconds.

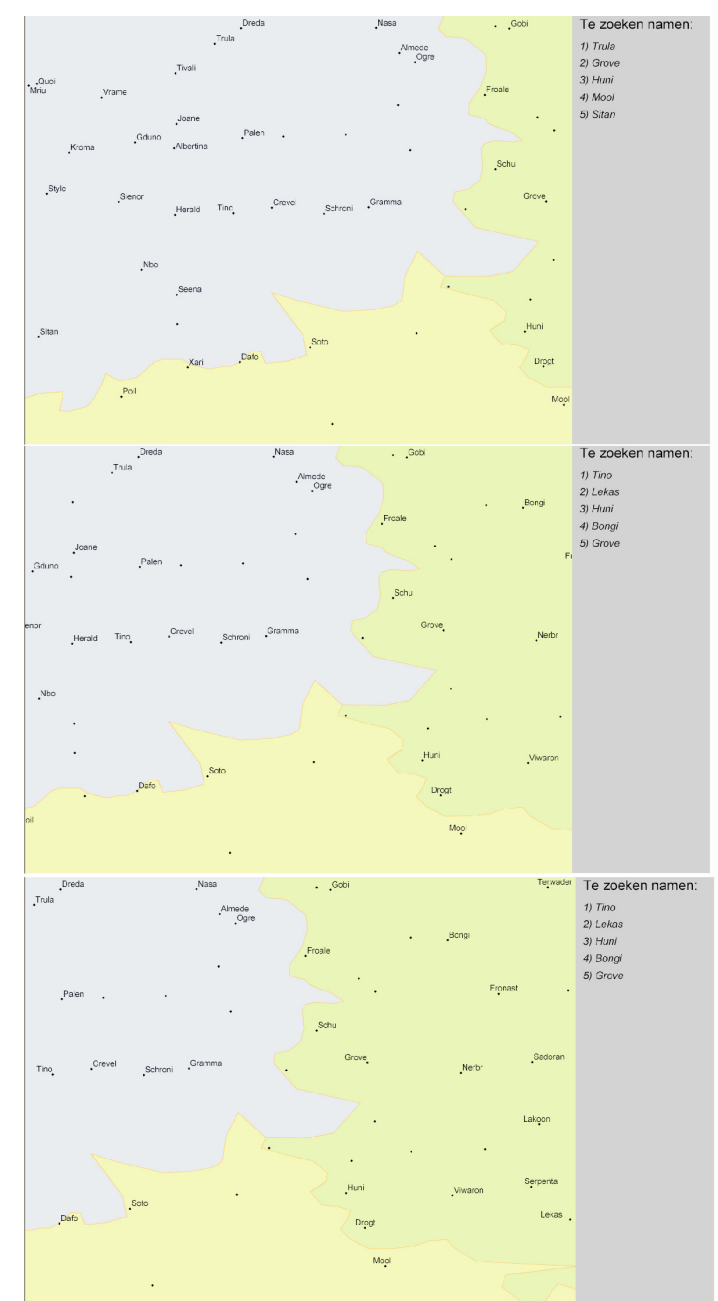

Figure 2. Illustration of the three subsequent intervals of the map stimulus (top: initial view; middle: a view during the translation; bottom: final view)

On the right side of each map stimulus a list of five names that were visible during the whole trial. The content of this list changed after the interaction: three names remained in the list and two were new ones. Due to the interaction, the absolute position of all labels on the map changed: they moved horizontally to the left over a certain fixed distance (the pan distance). In the list itself, the three names that were retained were also displayed on a different position after the interaction.

During the study, each participant had to repeat a number of tasks on each of the 20 map stimuli. First, he had to locate the five names in the initial view. Each time the participant found a name he had to push a button, resulting in a response time measurement. In order to avoid distracting the participant from the actual task, he had to push the same button for each label. After 50 seconds, the horizontal pan operation was simulated, during which the current view and 
the list with names changed. Next, the participant again had to locate these five names in the new view and push a button each time he found a name. When the participant had located all names, he could terminate the current trial himself and proceed to the next map.

The instructions were read out loud to each of the participants to make sure that everybody got them in exactly the same way. They contained a detailed description of the structure of the study, including how many names they had to find, the number of trials, duration of the initial view, the pan operation, and a description of the equipment. Furthermore, each user had to do two demo-trials before starting with the actual test. The goal of these demo-trials was to make the participant familiar with the task, stimuli and equipment. As a consequence, nothing was recorded during the demo-trials: the participant could still make misyakes or ask additional questions.

The task described above corresponds to a visual search. This is a task which is rather often executed on a map: the user wants to locate multiple points of interest (POIs) on a map, such as tourist attractions or cities. Phillips et al. (1978) also considered searching to be a good task to evaluate a map. They used the time to find a name as a measure to evaluate different types of typography used on a map. The disadvantage of working with a map on a relatively small screen is that these POIs are not situated within the extent of one view (with a certain scale). The user has to execute a pan operation to get both objects within one view. Consequently, he has to relocate the POIs from the previous view and the POIs in the new view.

In order to obtain a similar effect in the controlled environment of the experiment, it was decided to list five names, from which three were repeated after the interaction. This also prevented the participant from tracking the relative position of the labels during the simulated pan operation. Since the names which remained in the list were chosen randomly, the user could not know beforehand which label he would have to relocate after the interaction. Otherwise, the results would not have been comparable between the different maps. Otherwise, the results would not have been comparable between the different maps. In order to keep the participant's search behaviour as natural as possible, he could choose whether he wanted to search for the five names in the given order or randomly. As can be derived from Figure 1, the difference between the two algorithms is situated in the overlap zone. As a consequence, after the interaction, the user has to relocate the majority of the names in this part of the map.

Figure 3 gives an overview of the different types of cognitive processes that could be identified during this visual search on the map. When the map stimulus is displayed the participant will read one or more names from the list displayed on the right side of the map. Then he will look at the map to initiate the search. If the map is displayed for the first time, he will need some extra time to orientate it. During his search, he will try to identify potential label candidates: labels which look like the one he had read form the list earlier (e.g., length, shape of subsequent characters). When he has identified such a potential candidate, the participant will compare it with the information stored in his memory. If he finds a match he can either return to the list with names to read one or more new ones or, if he still has one or more names in memory, he will start searching again on the map. If no match was found, he will continue 
searching on the map. A more detailed discussion about these cognitive processes and their relation to the Cognitive Load Theory can be found in Ooms et al. (2012b).

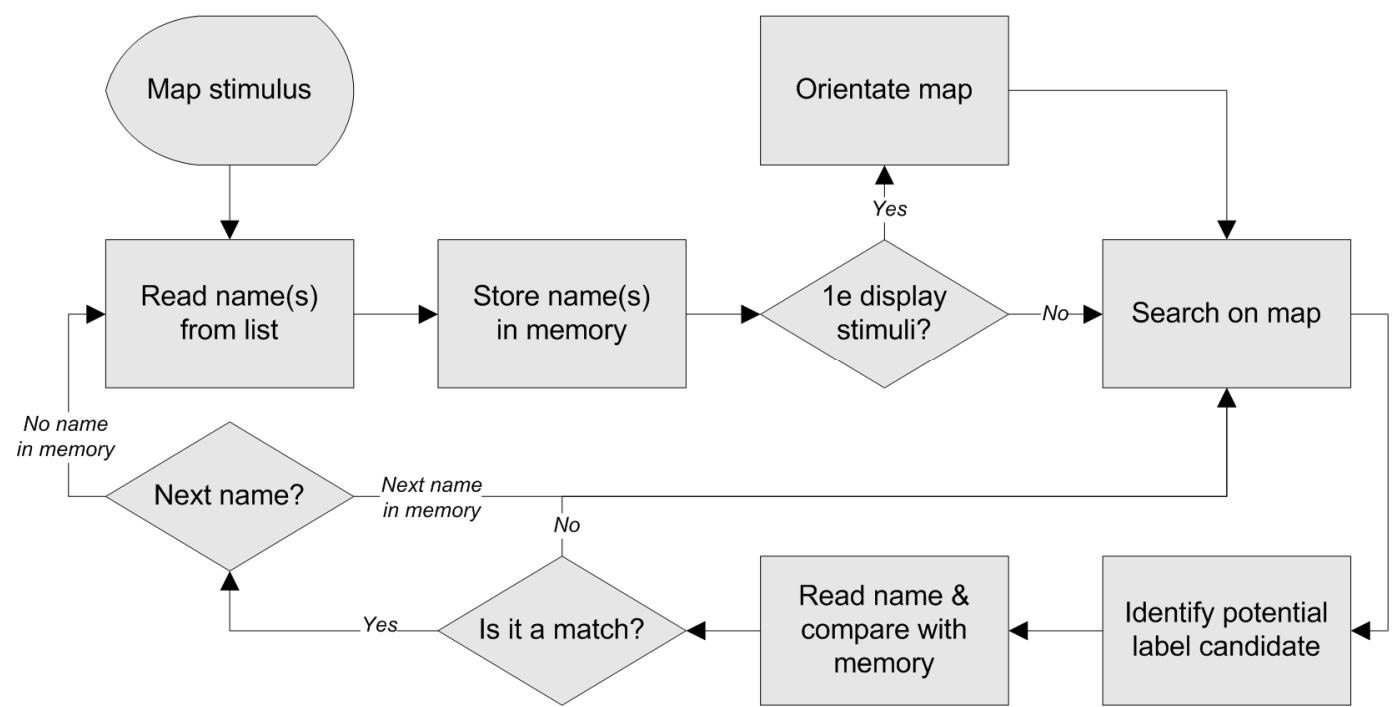

Figure 3. Overview of the users' cognitive processes during the visual search task

After completing the 20 trials, a questionnaire was presented that gathered background information, structured in several themes: general information, known regions, familiarity with web maps, and feedback. The measurements from the tests could be distorted by certain factors such as the familiarity of a participant with a certain region displayed during the tests. The questionnaire is thus as a tool to eliminate biases and explain these possible distortions in the data.

\section{Apparatus and data}

The Eye Tracking laboratory of the Department of Experimental Psychology (Ghent University, Belgium) is equipped with an Eye Link 1000 device. This eye tracking device from SR Research (Mississauga, Ontario, Canada) can sample a person's Point Of Regard at a rate of 1,000 Hz (or once every millisecond). The eye movements are recorded from one eye only. The software used to program the study and subsequently record the eye movements is the Experiment Builder (SR Research). Data Viewer software (SR Research) is used to conduct the initial processing and visualization of the obtained raw eye movement data. This software also allows identifying eye movement metrics related to fixation and saccades. The monitor on which the stimuli are presented has a size of 21 inch and a resolution of 1280x1024pixels.

During the study, a number of different elements were recorded simultaneously, which have to be combined in a correct way. First, response times were measured related to the moment a participant pressed a button indicating that he had found a label in the current view. A comparison of these response time measurements between different map designs can then indicate which design allows a faster retrieval of information. 
However, when using this method alone, there is absolutely no information on which labels are found or whether the participant found the correct labels. Furthermore, there is no indication of how easily the information on the map can be processed by the user. This type of information can typically be derived from the eye movement metrics related to the fixations. The duration of the fixation, for example, gives an indication of the time a user needs to process a certain view. However, eye movement data alone do not allow detecting if a label is found by a user and if it is the correct label. The user can 'accidently' overlook the label without actually identifying it as one of the labels from the list.

Only the combined data from the button actions and the eye movements allow identifying whether a label is found by the user and which label this is. The time measurements from the button actions thus indicate when a user finds a label; the location of the fixation at that moment identifies the label that was found. But caution is needed when combining measurements from different sources as it may cause synchronization errors. Synchronicity is achieved during this study because all recordings (eye movements and response time measurements) were processed simultaneously by one software system.

To get insights in the map users' cognitive processes during this task, data from multiple different sources were analysed, providing information about different aspects of a user's cognitive processes and attentive behaviour. Response time measurements are a type of performance measurement which is very often used in the field of human-computer interaction to evaluate the design of, among others, user interfaces and web pages (Nielsen, 1993). In this case, these measurements indicate how fast a user can find a name on a map. A more effective map design would result in smaller response times because the user can process the (visual) information more easily (Harrower, 2007; Phillips, et al., 1978).

More detailed information about the participants' cognitive processes can be derived from the eye movement metrics. The work of Jacob and Karn (2003) and Poole and Ball (2006) provide an excellent overview of a wide range of studies in which the eye movements of participants are analysed, which eye movement metrics are most often used, and what can be derived from them in terms of human-computer interaction. Since the goal of this research is to test the effectiveness of two different map designs (that are a result of two different label placement algorithms), the participants' fixations are analysed: number of fixations and fixation duration. For example, the duration of the fixations may give insights in the difficulty with which a user processes the visual information presented to him. A very chaotic, and thus not very effective, map would consequently result in longer fixation durations (Jacob \& Karn, 2003; Poole \& Ball, 2006).

As mentioned before, a close link between a participants eye movements and his attentive behaviour can be assumed if complex cognitive processes are triggered (corresponding to a high cognitive load). The task and the stimuli described above might seem rather simple, but they do trigger a high cognitive load: while the participant is searching for a label on the map, he is also trying to remember the locations of the names located previously. This results in an increasing cognitive load (Ooms et al., 2012b). Furthermore, the basic design of the maps and the 
controlled laboratory environment prevents the participants to get distracted, both due to internal (within the task or stimuli) or external (e.g., noises, striking elements in the surroundings) causes. Based on the considerations mentioned above, it can safely be assumed that the participants will not be distracted during the study and consequently, that there is a close link between the eye movements and his attentive behaviour. Hence, the metrics resulting from the eye moment study can be used to obtain insights in the underlying cognitive processes.

A qualitative, visual analysis of the eye movements also allows analysing the spatial dimension of the obtained data. Deviations or patterns in the users' scanpaths, a succession of subsequent fixations and saccades, give insights in the users' search behaviour and might indicate problems in the (map) design (Ooms, et al., 2012a). Finally, these quantitative data sources are complemented with the results of a post study questionnaire. The goal of this questionnaire is to obtain information about the users' preferences towards a certain type of label placement. A user's preference towards a certain type of design might not always correspond to the most effective one (Nielsen, 1993).

\section{RESULTS \& DISCUSSION}

The results from two participants were removed from the dataset. The first participant was familiar with the presented regions and knew some of the names by heart. The other participant was extremely slow in locating the names. As a consequence, he could not execute the assignment correctly. From the remaining participants, all measurements related to locating a wrong name were removed from the dataset. Analyses of variance (ANOVAs) were performed across participants with map design (border-labels relocated vs. all-labels relocated) as independent variables. The dependent variables were the mean reaction times across trials, the fixation count, and the fixation durations.

\section{Relative response times}

The results showed no difference between the border-labels relocated and all-labels relocated map design conditions $(F=.069, P=.793)$. The mean reaction time for locating the ten labels was $5614 \mathrm{~ms}$ ( $S D=1716 \mathrm{~ms}$ ) for the condition in which only labels at the border were relocated after an interaction and $5570 \mathrm{~ms}(S D=1630 \mathrm{~ms})$ for the condition in which all labels could be relocated after the interaction. The mean values (in $\mathrm{ms}$ ) for locating the different labels in the two map designs are illustrated in Figure 4. In the map design Border only labels near the border could be relocated after the interaction; in map design Total all label could be relocated. Five labels were located before the simulated interaction (Before) and five after this interaction (After). Paired comparisons of the intervals of locating each label in both map designs (e.g., the first label that was found in the Border-design vs. the Total-design, the second label that was found in the Border-design vs. the Total-design) showed no significant differences for any of the intervals $(P s>.106)$.

When a map is displayed to the user for the first time he has to orientate it. The time needed to do this is included in the time interval to locate the first label. Since no difference was 
found in this first time interval, it can be concluded that there was no difference in how fast the user can orientate the map. After the simulated pan operation, the user will orientate the map again: he wants to link the initial view with the map view after the interaction. Again, the orientation time is included in the time interval needed to locate the first label. Also in this case, no significant different could be detected between the two map designs (or label placement methods). As mentioned previously, users might be distracted after the simulated pan operation if some labels' relative position suddenly changes after the interaction. However, in the case of the proposed label placement algorithm, no significant improvement could be detected. Another effect of the proposed algorithm on the map design is the lower quality in the final label placement: fewer labels are placed in their most preferred position. Since no significant difference could be detected in the corresponding response times of the two types of map design, it can be concluded that the users were not negatively affected in their visual search due to this lower quality.
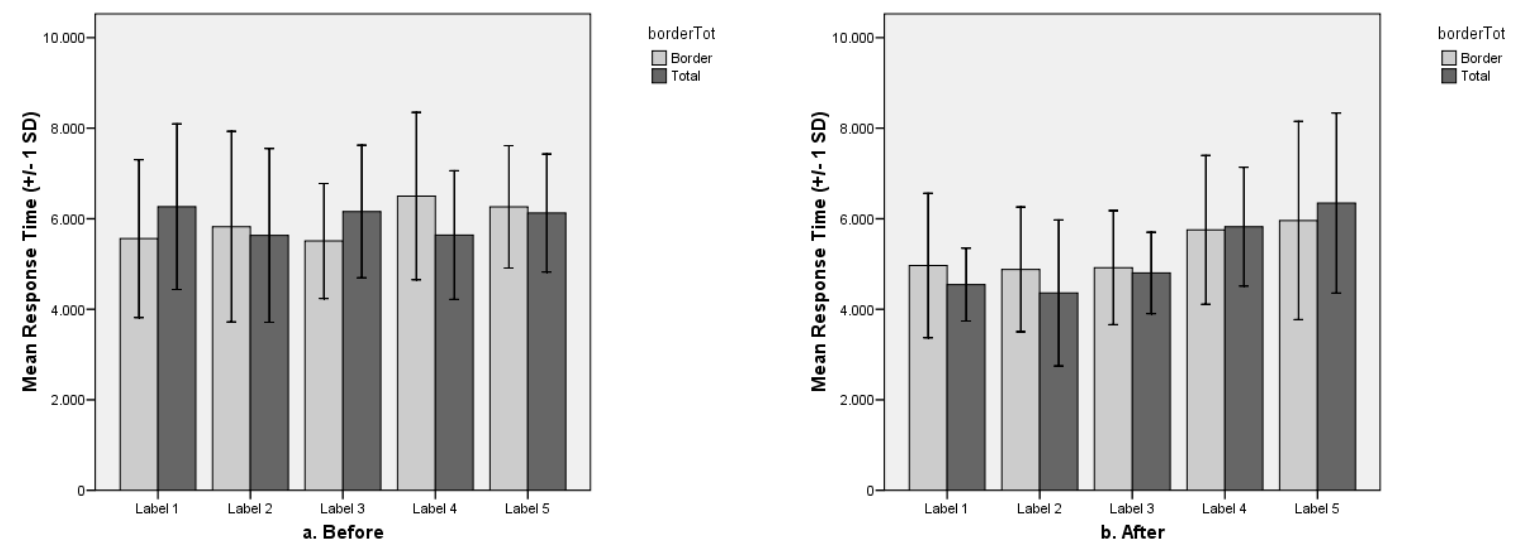

Figure 4. Mean response time measurements (ms, +/- 1SD) for both types of label placement related to finding the five labels, before (a) and after (b) the interaction

\section{Fixation count and duration}

The fixation count corresponds to the number of fixations during the trial. Since this is inherently related to the length of the trial, a more objective metric is used: number of fixations per second. The number of fixations per second is closely linked with a second metric often used in eye movement research: fixation duration. Although both measurements are linked with each other, it might be very useful to consider them both in order to obtain a better understanding in the users' cognitive processes. This duration of a fixation may give an indication about how the user processes the (visual) information presented to him. Longer fixation durations may indicate that the user is confused by or has difficulty in reading the display, but it could also indicate that the user find that part of the display interesting (Poole \& Ball, 2006). However, the basic structure of the map stimuli in the experiment assures that all regions on the maps look equally interesting. Therefore, the latter explanation is not considered in this study. If the labels are placed in a very 
chaotic way on the map it is expected that the user needs more time to process the visual stimuli, resulting in longer fixation durations. Some of the labels on a map with a Border-design, are placed in a less preferred position than with a Total-design. Therefore, it might be expected that the proposed algorithm creates maps that are more difficult to process, resulting in deviating eye movement metrics such as longer fixation durations.

The mean number of fixation per second (fix/s) for both map designs is 3.843 (SD: .436) with a minimum and maximum of respectively 2.425 and $5.036 \mathrm{fix} / \mathrm{s}$. The mean number of fix/s counts 3.829 for the Border-map design (SD: .451) and 3.856 (SD: .422) for the Total-map design. A one way ANOVA over all response times and with map design as a factor shows no significant difference on the level of the fixed factor $(F=.822 ; P=.365>.05)$. Since the map design type is related to how the labels are placed after an interaction, the label's positions might have a more pronounced influence on the user after the interaction. As a consequence, the dataset is further divided into two subsets: before and after the interaction. The mean fixation counts, related to both map designs, are illustrated in Figure 5. In this case, it can be noticed that both mean values are smaller after the interaction and that the difference is larger with a Border-map design. However, a paired comparison between both map designs shows no significant difference in the results, both Before $(F=.118, P=.731)$ and After $(F=.787, P=.376)$.

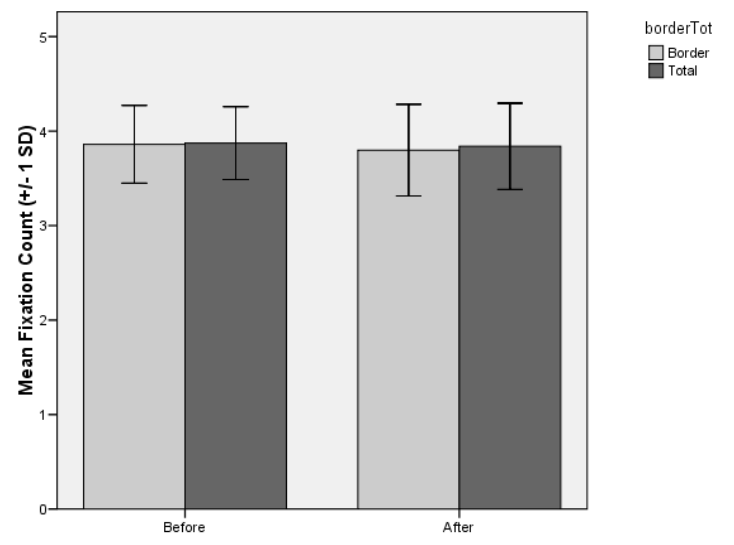

Figure 5. Mean fixation count (fix/s, +/- 1SD) for both types of label placement, before and after the interaction

The mean duration of fixations is $228.2 \mathrm{~ms}$ ( $S D: 32.8 \mathrm{~ms}$ ) for both maps designs, with a minimum of $164.3 \mathrm{~ms}$ and a maximum of $357.2 \mathrm{~ms}$. When only considering the Border-map design this mean duration is $229.6 \mathrm{~ms}$ ( $S D: 34.2 \mathrm{~ms}$ ), whereas this is $226.9 \mathrm{~ms}$ ( $S D: 31.5 \mathrm{~ms}$ ) for the Total-map design. Also on this dataset, a one way ANOVA is applied which results correspond to these from the fixation count: no significant difference on the level of the independent factor $(F=1.439 ; P=.231>.05)$. The mean fixation durations, related to if they occurred before or after the interaction, are presented in Figure 6. Also in this case, the paired comparison showed no significant difference between the two map designs, both Before $(F=.304, P=.582)$ and $\operatorname{After}(F=1.228, P=.268)$. 


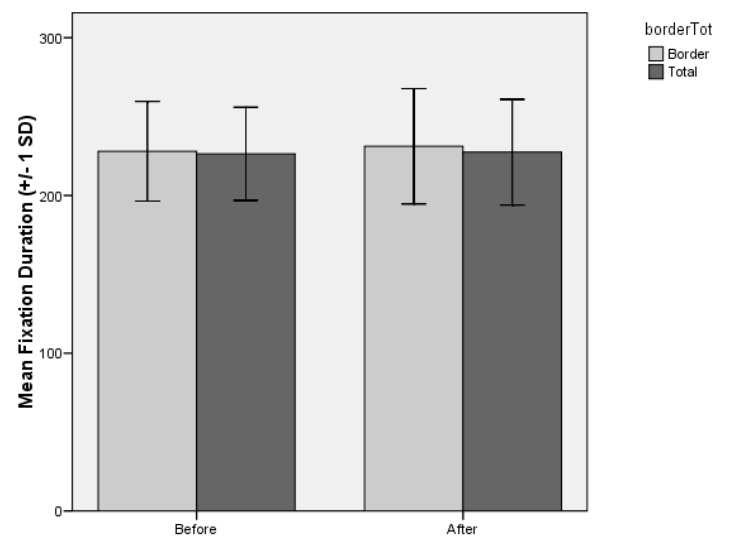

Figure 6. Mean fixation duration (ms, +/- 1SD) for both types of label placement, before and after the interaction

The eye movement metrics thus show no significant deviations between the application of the two different label placement methods. This means that, although fewer labels are placed in a more preferred position by the proposed algorithm, the users do not have more difficulty to process the information. This may be explained by the fact that it only concerns a limited number of labels that are not placed in a more optimal position: certain labels that were located near the border of the previous view and were therefore restricted in their placement.

\section{Qualitative scanpath analysis}

Besides the statistical analysis of the eye movement metrics, interesting elements regarding the users search behaviour might be obtained from a visual analysis of the scanpaths. These scanpaths are a succession of subsequent fixations and saccades. The spatial distribution of these scanpaths was analysed and compared using the Visual Analytics Toolkit, whose functions - and their applicability on eye movement data - are described in the work of Ooms et al. (2011). The scanpaths were filtered based on their attributes, using a fixed time interval. The distribution of the scanpaths related to the same area, but with the application of a different label placement algorithm, could thus be compared.

In order to create more comparable datasets, the eye movements of a certain map were filtered based on a fixed time interval. This is necessary because the users could end the trial themselves when they had located all labels. As a consequence, each trial had a different length. Since we had already fixed the time interval before the interaction at 50s, the same will be done to visualise the scanpaths after the interaction.

A comparison of the scanpaths of the corresponding map designs, both before and after the interaction, reveals deviations or differences in how the users search on the map. For every combination, the scanpaths of the users were very similar. As an example, these scanpaths are illustrated for one of the maps in Figure 7. The two upper pictures correspond to maps which are created with a traditional labels placement algorithm (Total-map design), whereas the two lower pictures are created with the proposed algorithm (Border-map design). The pictures on the left 
side illustrate the scanpaths before the interaction and the pictures on the right side the ones after the interaction (during 50s).
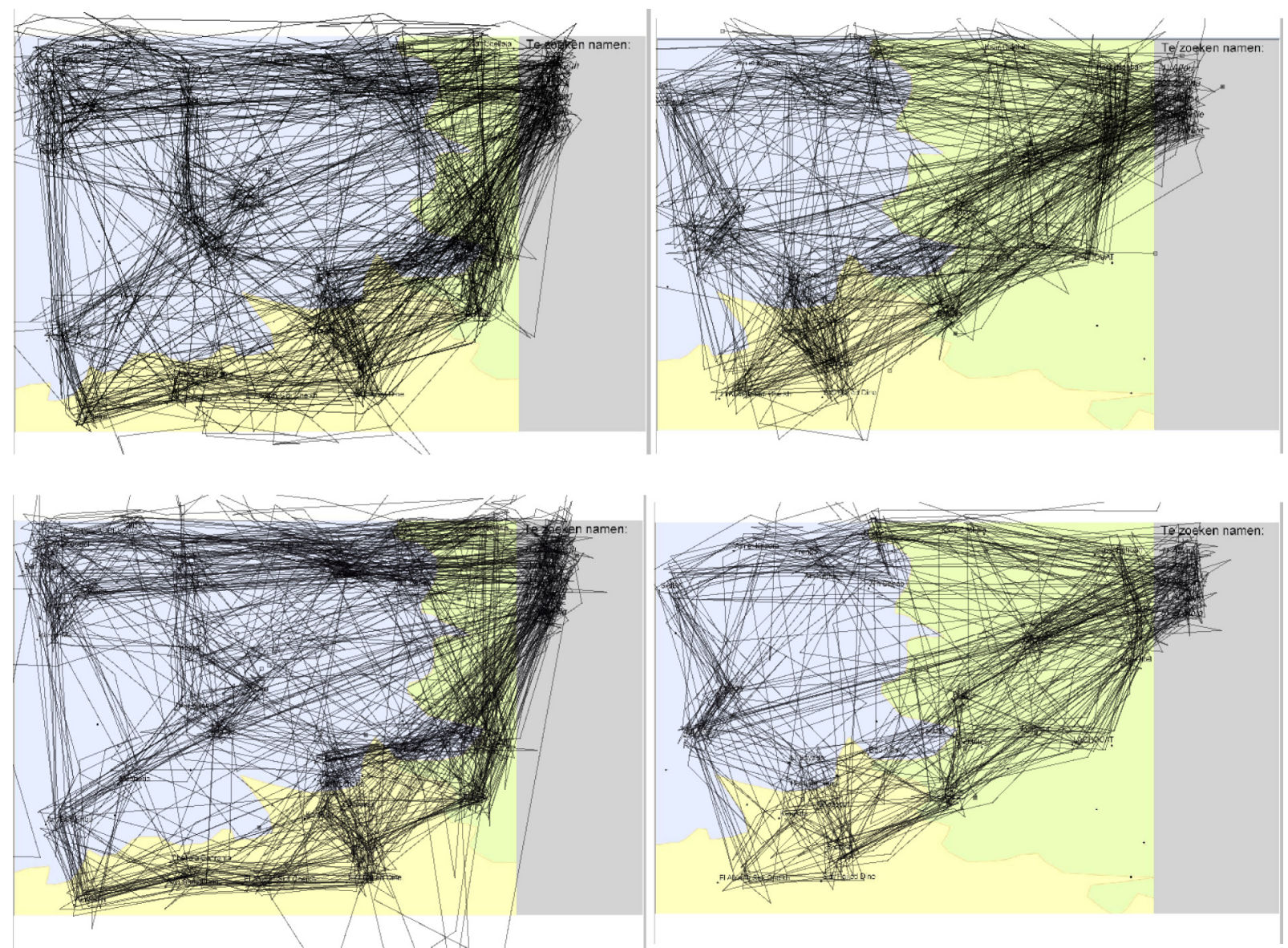

Figure 7. The users' scanpaths when searching on one of the maps (top: Total-map design, bottom: Border-map design; left: before the interaction, right: after the interaction)

\section{Questionnaire}

A number of questions in the post study questionnaire were related to the two map designs presented to each participant. The goal of these questions was to discover whether the preference of the users towards one label placement type corresponds to the results of the statistical comparisons: time measurements and eye movement metrics. These questions were formulated as follows (translated from Dutch):

During the study, two types of maps were presented to you.

- Describe the difference between both map types.

- Which of the two types do you prefer the most?

- Why do you prefer this type more than the other one?

All of the participants answered to the first question that they did not notice any difference between the 20 maps presented to them. Consequently, no useful answers could be 
derived from the two subsequent questions. But the answers on the first question clearly indicate that the participants did not notice the difference between the maps (or label layouts) presented to them.

\section{CONCLUSION \& FUTURE WORK}

This paper describes a study in which the effectiveness of an improved label placement algorithm (in terms of efficiency) towards the user is investigated. The combination of response time measurements and eye movement metrics allows identifying when a label was found and which label was found by the user. The gathered eye movement metrics, duration and number of fixations, allow detecting whether the different label layouts have an influence on the user's cognitive processes and thus on its effectiveness towards the user.

The analysis of the participant's response times shows that the proposed label placement algorithm does not have any significant influence on the user's cognitive processes. The assignment can be completed in the same time interval on both map designs, which means that the different label layout does not affect the participant his orientation process, nor the his visual search on the map. More detailed analyses of the user's cognitive processes, using the eye movement metrics fixation count and fixation duration, confirm this finding even further. Both the number of fixations per seconds and the duration of these fixations are not significantly different. Furthermore, no deviations in the users' search behaviour could be derived from the visual analysis of the scanpaths. The questionnaire also revealed that the participants did not notice the difference between both label placement methods. It can thus be concluded that the two map designs, with or without the application of the proposed algorithm, are as difficult to interpret by the participants. Although both map designs created different visual outputs, the participants stated that they did not notice any difference between the maps presented to them. This confirms that the application of the more efficient algorithm does not influence the user in any way. The label placement algorithm is thus more efficient without affecting the user and thus the effectiveness of the map in any way.

However, these results were obtained during a controlled study, using a basic map design, a simple task and in a laboratory environment, which is different from a real life situation. So the question arises: how generalisable are these results? During the controlled study, the goal was to direct the focus of the participants only on the labels on the maps, avoiding other influencing or distracting factors (such as other map elements) as much as possible. As a consequence, if there would have been any effect of this adapted label placement algorithm on the map user, it would be expected to be the clearest in this study. More complex maps would lessen the effect of the lower qualitative label placement, as there are too many other elements on the map to which the focus of the user will be directed. Nevertheless, it should also be noted that the actual labels will be placed differently due to the other map elements, which may also affect the quality of the placement and thus its efficiency towards the user. In order to be able to answer this question objectively, a series of follow-up studies have to be conducted in which more complex map 
stimuli are used. Gradually adding more map elements to the stimuli would allow investigating which elements (or what level of complexity) would have to greatest impact on the map user.

In order to be able to create more effective maps, more information is needed on how users actually interpret maps: how do they orientate a map, how do they process, store, and retrieve the visual information presented to them. Detailed insights in the user's cognitive structures are thus essential to understand these processes, especially while working with highly interactive and dynamic maps. Eye tracking is an excellent method to get in touch with the user's cognitive processes while working with visual stimuli. More detailed studies are planned to investigate how users orientate these dynamic maps, how their cognitive maps are created and what influences them.

\section{REFERENCES}

Ahn, J., \& Freeman, H., 1984. A Program for Automatic Name Placement. Cartographica, 21(2\&3), 101-109.

Barrault, M., 1998. Le Placement Cartographique des Écritures : Résolution d'un problème à Forte Combinatoire et Présentant un Grand Nombre de Constraintes Variéees, University of Marne-la-vallée, France.

Barrault, M., 2001. A Methodology for Placement and Evaluation of Area Map Labels. Computers, Environment and Urban Systems, 25(1), 33-52.

Been, K., Daiches, E., \& Yap, C., 2006. Dynamic Map Labeling. IEEE Transactions on visualization and computer graphics, 12(5), 773-780.

Bradstreet, L., Barone, L., \& While, L., 2005. Map-labelling with a Multi-Objective Evolutionary Algorithm. Proceedings, Papers from the 2005 Conference on Genetic and Evolutionary Computation, Washington: 1937 - 1944.

Brodersen, L., Andersen, J. H. K., \& Weber, S., 2001. Applying the Eye-Movement Tracking for the Study of Map Perception and Map Design. Kort and Matrikelstyrelsen. National Survey and Cadastre Denmark, Copenhagen, Denmark.

Chazelle, B., \& 36 co-authors, 1996. Application Challenges to Computational Geometry. Report TR-521-96. Computational Geometry Impact Task Force's report: Princeton CS Dept. Technical. Princeton University, Princeton NJ.

Christensen, J., Marks, J., \& Shieber, S., 1995. An Empirical Study of Algorithms for PointFeature Label Placement. ACM Transactions on Graphics, 14(3), 203-232.

Coltekin, A., Heil, B., Garlandini, S., \& Fabrikant, S. I., 2009. Evaluating the Effectiveness of Interactive Map Interface Designs: a Case Study Integrating Usability Metrics with EyeMovement Analysis. Cartography and Geographic Information Science, 36(1), 5-17.

Cravo, G. L., Ribeiro, G. M., \& Lorena, L. A. N., 2008. A Greedy Randomized Adaptive Search Procedure for the Point-Feature Cartographic Label Placement. Computers \& Geoscience, 34(4), 373-386.

Cuenin, R., 1972. Cartographie Générale, Eyrolles, Paris.

Djamasbi, S., Siegel, M., \& Tullis, T., 2010. Generation Y, Web Design, and Eye Tracking. International Journal of Human-Computer Studies, 68(5), 307-323.

Downs, R. M., \& Stea, D., 1977. Maps in Minds. Reflection on Cognitive Mapping. Harper \& Row, New York.

Duchowski, A. T., 2007. Eye Tracking Methodology - Theory and Practice, Springer, London. 
Edmondson, S., Christensen, J., Marks, J., \& Shieber, S., 1996. A General Cartographic Labeling Algorithm. Cartographica, 33(4), 13-23.

Fabrikant, S. I., Rebich-Hespanha, S., Andrienko, N., Andrienko, G., \& Montello, D. R., 2008. Novel Method to Measure Inference Affordance in Static Small-Multiple Map Displays Representing Dynamic Processes. The Cartographic Journal, 45(3), 201-215.

Fleetwood, M. D., \& Byrne, M. D., 2006. Modeling The Visual Search of Displays: a Revised Act-R Model of Icon Search Based on Eye-Tracking Data. Human-Computer Interaction, 21(2), 153-197.

Freeman, H., 2005. Automated Cartographic Text Placement. Pattern Recognition Letters, 26(3), 287-297.

Haklay, M., Singleton, A., \& Parker, C., 2008. Web Mapping 2.0: The Neogeography of the GeoWeb. Geography Compass, 2(6), 2011-2039.

Harrower, M., 2007. The Cognitive Limits of Animated Maps. Cartographica, 42(4), 349-357.

Henderson, J. M., \& Hollingworth, A., 1998. Eye Movements During Scene Viewing: an Overview. In: G. Underwood (Ed.), Eye Guidance in Reading and Scene Perception, Elsevier, Oxford, pp. 49-65.

Imhof, E., 1975. Positioning Names on Maps. The American Cartographer, 2(2), 128-144.

Jacob, R., \& Karn, K., 2003. Eye Tracking in Human-Computer Interaction and Usability Research: Ready to Deliver the Promises. In: R. Radach, J. Hyona \& H. Deubel (Eds.), The Mind's Eye: Cognitive and Applied Aspects of Eye Movement Research, Elsevier, Amsterdam, pp. 573-605.

Joachims, T., Granka, L., \& Pan, B., 2005. Accurately Interpreting Clickthrough Data as Implicit Feedback. Proceedings, 28th Annual International ACM SIGIR Conference on Research and Development in Information Retrieval, Salvador, Brazil: 154 -161.

Jobst, M., \& Döllner, J., 2008. Neo-Cartographic Influence on Map Communication in LBS. In: G. Gartner \& K. Rehrl (Eds.), Lecture Notes in Geoinformation and Cartography (Vol. Location Based Services and TeleCartography II), Springer, Heidelberg, Berlin, pp. 207-219.

Kakoulis, K. G., \& Tollis, I. G., 2001. On the Complexity of the Edge Label Placement Problem. Computational Geometry. Theory and Applications, 18(1), 1-17.

Kraak, M.-J., \& He, N., 2009. Organizing the Neo-Geography Collections with Annotated Space-Time Paths. Proceedings, 24th International Cartographic Conference, Santiago, Chile.

Marks, J., \& Shieber, S., 1991. The Computational Complexity of Cartographic Label Placement, TR-05-91, Center for Computing Technology, Harvard.

Montello, D. R., 2002. Cognitive Map-Design Research in the Twentieth Century: Theoretical and Empirical Approaches. Cartography and Geographic Information Science, 29(3), 283-304.

Montello, D. R., 2009. Cognitive Research in GIScience: Recent Achievements and Future Prospects. Geography Compass, 3(5), 1824-1840.

Mote, K. D., 2007. Fast Point-Feature Label Placement for Dynamic Visualizations. Information Visualization, 6(4), 249-260.

Nielsen, J., 1993. Usability Engineering. Morgan Kaufmann, San Francisco.

Nivala, A., 2007. Usability Perspectives for the Design of Interactive Maps. University of Technology, Helsinki. 
Ooms, K., Andrienko, G., Andrienko, N., De Maeyer, P., \& Fack, V., 2012a. Analysing the Spatial Dimension of Eye Movement Data Using a Visual Analytic Approach. Expert Systems with Applications, 39(1), 1324-1332.

Ooms, K., De Maeyer, P., Fack, V., Van Assche, E., \& Witlox, F., 2012b. Interpreting Maps Through the Eye of Expert and Novice Users. International Journal of Geographic Information Science. (accepted - in press)

Phillips, R. J., Noyes, E., \& Audley, E. J., 1978. Searching for Names on Maps. The Cartographic Journal, 15(2), 72-77.

Poole, A., \& Ball, L. J., 2006. Eye Tracking in Human Computer Interaction and Usability Research: Current Status and Future Prospects. In: C. Ghaoui (Ed.), Encyclopedia of Human Computer Interaction, Idea Group, Pennsylvania, pp. 211-219.

Rayner, K., 1998. Eye Movement in Reading and Information Processing: 20 Years of Research. Psychological Bulletin, 124(3), 372-422.

Rubin, J., \& Chisnell, D., 2008. Handbook of Usability Testing. How to Plan, Design and Conduct Effective Tests (second ed.), Wiley Publishing, Indianapolis.

Salvucci, D. D., \& Anderson, J. R., 2001. Automated Eye-Movement Protocol Analysis. Human-Computer Interaction, 16(1), 39-86.

Schiessl, M., Duda, S., Thölke, A., \& Fischer, R., 2003. Eye Tracking and its Application in Usability and Media Research. MMI-Interaktiv Journal - Online Zeitschrift zu Fragen der Mensch-Maschine-Interaktion, 6, 41-50.

Slocum, T. A., Blok, C., Jiang, B., Koussoulakou, A., Montello, D. R., Fuhrmann, S., \& Hedley, N. R., 2001. Cognitive and Usability Issues in Geovisualization. Cartography and Geographic Information Science, 28(1), 61-75.

Strijk, T., \& van Kreveld, M., 2002. Practical Extensions of Point Labeling in the Slider Model. GeoInformatica, 6(2), 181-197.

Turner, A. J., 2006. Introduction to Neogeography, O'Reilly Media, Sebastopol, CA.

van Dijk, S., 2001. Genetic Algorithms for Map Labeling. Utrecht University, Utrecht.

van Dijk, S., van Kreveld, M., \& Wolff, A., 2002. Towards an Evaluation of Quality for Names Placement Methods. International Journal of Geographical Information Systems, 16(7), 641-661.

van Elzakker, C., 2004. The Use of Maps in the Exploration of Geographic Data, Labor Grafimedia b.v., Utrecht.

van Kreveld, M., Strijk, T., \& Wolff, A., 1999. Point Labeling with Sliding Labels. Computational Geometry. Theory and Applications, 13(1), 21-47.

Wagner, F., Wolff, A., Kapoor, V., \& Strijk, T., 2001. Three Rules Suffice for Good Label Placement. Algorithmica, 30(2), 334-349.

Wolff, A., Knipping, L., van Kreveld, M., Strijk, T., \& Agarwal, P. K., 2000. A Simple and Efficient Algorithm for High-Quality Line Labeling. Proceedings, $7^{\text {th }}$ Annual Conference GIS Research UK, Southampton, UK.

Wood, C. H., 2000. A Descriptive and Illustrated Guide for Type Placement on Small Scale Maps. The Cartographic Journal, 37(1), 5-18.

Yamamoto, M., Camara, G., \& Lorena, L. A. N., 2002. Tabu Search Heuristic for Point-Feature Cartographic Label Placement. GeoInformatica, 6(1), 77-90.

Yamamoto, M., Camara, G., \& Lorena, L. A. N., 2005. Fast Point-Feature Label Placement Algorithm for Real Time Screen Maps. Proceedings, 7th Brazilian Symposium on GeoInformatics, São Paulo, Brazil: 122-138. 
Yoeli, P., 1972. The Logic of Automated Map Lettering. The Cartographic Journal, 9(2), 99108.

Zambarbieri, D., Carniglia, E., \& Robino, C., 2008. Eye Tracking Analysis in Reading Online Newspapers. Journal of Eye Movement Research, 2(4), 1-8.

Zhang, Q., \& Harrie, L., 2006. Real-Time Map Labelling for Mobile Applications. Computers, Environment and Urban Systems, 30(6), 773-783.

Zoraster, S., 1990. The Solution of Large 0-1 Integer Programming Problems Encountered in Automated Cartography. Operations Research, 38(5), 752-759.

Zoraster, S., 1997. Practical Results Using Simulated Annealing for Point Feature Label Placement. Cartography and GIS, 24(4), 228-238. 